\title{
The Use of Embedded Interaction Mechanisms for Low-Level Analysis Tasks
}

\author{
Kari Sandouka \\ Dakota State University \\ kari.sandouka@trojans.dsu.edu
}

\author{
Cherie Noteboom \\ Dakota State University \\ cherie.noteboom@dsu.edu
}

\begin{abstract}
The use of information visualization is a strategy to reduce information overload and cognitive efforts. Interaction mechanisms aid the exploration of data when it is not practical to display all data points in one visual display. This study reports the results of a pilot study. The purpose of the study is to determine what interactive mechanisms are used and how they support a task or set of tasks.
\end{abstract}

\section{Introduction}

The information visualization discipline provides solutions for decreasing information overload, aiding cognitive processing, and supporting sensemaking processes. The rate of data generation, collection, and storage of data hides valuable insights needed for these solutions. Value, through insight generation, is realized when individuals can make sense of the data. Information visualization tools combine the strength of computers with those of humans to create joint cognitive systems [1]. The purpose of these tools is to generate insight, not just visually display data [2].

Given the availability of data, visualization tools are being used to run businesses, solve problems, and aid decision-making processes [3, 4]. There are many unanswered questions regarding how the design of information visualizations are compatible with the way users think and reason [5]. There are three primary challenges for information visualization design. First, the ability to select the correct visual representation that users can understand and perceive. Second, conveying the information that the user needs. Lastly, providing the appropriate interaction mechanisms that allow effortless data exploration [6]. Not addressing these elements leads to ineffective visualization tools. An ineffective information visualization may cause pointless explorations, inaccurate or false knowledge, lost time, or lack of utilization due to frustration and confusion [7].

How a user interacts with the information visualization will impact insight generation, and ultimately, the effectiveness of the visualization tool [8]. Interaction is not simply a yes or no property of the tool; rather, there are several degrees determined by the extent of user engagement [9]. Despite the ubiquity of interactive visualizations, it remains a challenge to determine when interaction is appropriate and what mechanisms to use [10]. We view this as a gap between theory and practical use.

Designers know what is available for implementation, but do not always consider what users will need or what users will use to complete tasks. End-users often have difficulty interacting with information visualizations because of the 'Gulf of Execution [11, 12].' The gulf indicates a difference between what the user intends to do and the allowable actions supporting that intent; otherwise describing what a user needs to do and how do they do it. We report a user study conducted to investigate the use of interactions embedded in information visualizations. We explore the question: what interaction mechanisms are used to complete a given task or set of tasks? We report results from a pilot study that asks users to identify the interaction mechanisms they employed while using information visualizations.

\section{Related work}

The theoretical foundation of this investigation is a Human-Information Interaction (HII) framework by Sedig and colleagues $[10,13]$. The framework characterizes cognitive activities at four levels of granularity (see Figure 1). Events or actions are the lowest level and represent physical actions. Interactions build from events and consists of the actions performed on the visualization, along with subsequent reactions. Tasks are goal-oriented behaviors that provide the purpose for interacting and with information visualizations. Activities and tasks may be composed of sub-activities and sub-tasks (respective to the level). The study uses the bottom-up approach for applying the framework. Through this approach, the performance of a task gradually emerges over time through a sequence of interactions that a user performs [13]. 
The framework conceptualizes information visualization as a system, where the tool is the sum of all parts. Individual pieces generate properties that work cohesively with each other through relationships. The investigation focuses on the interaction level of abstraction, which lies between events and tasks. Interactions are composed of three elements: the human, the visualization, and a mediating computing device [11].

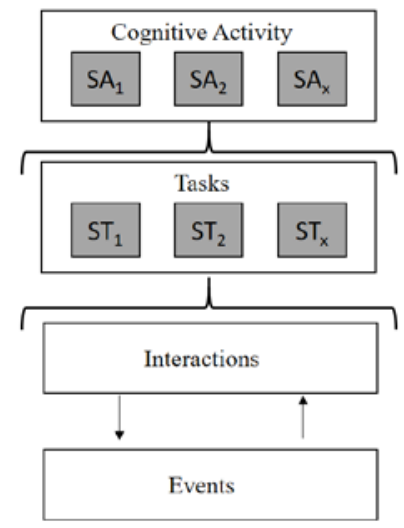

Figure 1. HII Levels of Abstraction for Cognitive Activities (adapted from [13])

The framework works in concert with extant research. The most common definition for information visualization is "the use of computer-supported interactive visual representations of data to amplify cognition [3].” Two foundational models emerged from this definition: the reference model for visualization [3] and the simple visualization model [14] (see Figure 2). These models show that insights are generated as the human participates in a feedback loop between reading the data displayed and interacting with the visualization. The feedback loop represents a discourse or dialog that is created and facilitated by interactions. The ' $E$ ' in the Simple visualization model stands for exploration. As a user perceives the Image, they generate knowledge. The user can choose to explore the data further by changing the specification creating the visual representation. The changes in specification update the visual representation, creating a dialog with the user. This loop continues as long as the user initiates changes.

The coupling between interactions and visual representations is a critical element to information visualization. For users to complete a task, they should be able to execute their intent. The design of an interactive visualization can be easy to use and easy to learn, but will always be dependent on the user [15].

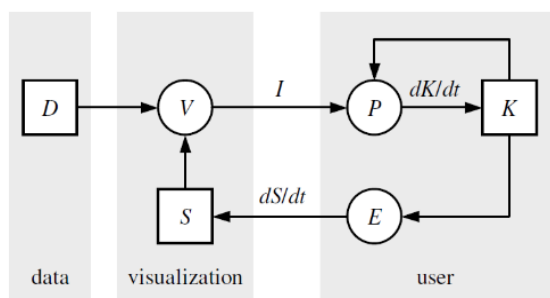

Figure 2. Simple Visualization Model (adapted from [14])

The gulf of execution develops when a user forms an intention and knows what action needs to take place but cannot execute the action [11, 12]. Implementing interactions involves a delicate balance: providing too many interaction mechanisms may cause cognitive overload or become time-intensive for the user. Providing too few interaction mechanisms provides a lack of possibilities leading to an inefficient and ineffective tool [10].

\subsection{Interactions}

Interactions are powerful tools that enable visual exploration and insight generation. Interaction mechanisms are features the individual apply to manipulate and interpret information visualizations, triggering feedback loops [16]. The interactive dialog between a visualization and the user creates a reciprocal relationship, fulfilling the purpose of the system [17]. Extant research provides several taxonomies for interaction mechanisms (see Table 1). While we know what mechanisms are available, little is known about how these mechanisms directly support analysis tasks nor what benefits the mechanisms provide.

Visualization designers must understand the intent of using the tool. Along with identifying these tasks (or intents), designers must understand how interaction mechanisms assist users in executing their tasks. These mechanisms are integral in developing the feedback loops that engage the user, allowing for the visualization to leverage the human perceptual system $[2,5]$.

\section{Study design}

To improve the understanding of what interaction mechanisms are used to support analysis tasks and bridge the gulf of execution, we conducted a study focusing on embedded interactions. The objective of the study is to determine what interaction mechanisms are used for a given task or set of tasks. The investigation is premised by the idea that designers 
Table 1. Common Interaction Mechanisms

\begin{tabular}{|c|c|c|c|c|c|c|c|c|}
\hline \multirow{2}{*}{\multicolumn{2}{|c|}{ Interaction Mechanisms }} & \multirow{3}{*}{ 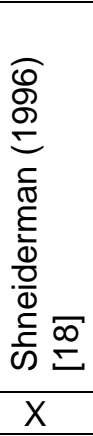 } & \multirow{3}{*}{ 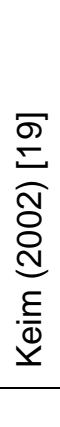 } & \multirow{3}{*}{ 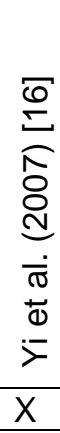 } & \multirow{3}{*}{ 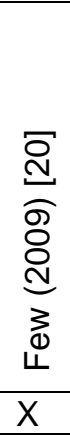 } & \multirow{3}{*}{ 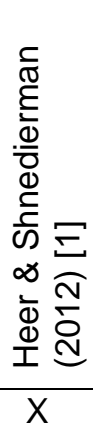 } & \multirow{3}{*}{ 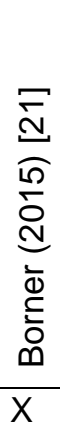 } & \multirow{3}{*}{ 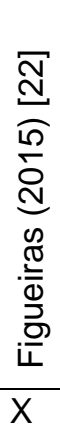 } \\
\hline & & & & & & & & \\
\hline Overview & entire data collection & & & & & & & \\
\hline Zoom & $\begin{array}{l}\text { scale visualization to view a } \\
\text { specific subset }\end{array}$ & $\mathrm{X}$ & $\mathrm{X}$ & & $\mathrm{X}$ & & $\mathrm{X}$ & $\mathrm{X}$ \\
\hline Filter & $\begin{array}{l}\text { reduce size of search, hide } \\
\text { data points conditionally }\end{array}$ & $\mathrm{X}$ & $\mathrm{X}$ & $\mathrm{X}$ & $\mathrm{X}$ & & $X$ & $\mathrm{X}$ \\
\hline $\begin{array}{l}\text { Details on } \\
\text { Demand }\end{array}$ & select an item to get details & $\mathrm{X}$ & & $\mathrm{X}$ & & $\mathrm{X}$ & & \\
\hline Relate & view relationships among items & & $\mathrm{X}$ & $\mathrm{X}$ & & $\mathrm{X}$ & $\mathrm{X}$ & $\mathrm{X}$ \\
\hline History & $\begin{array}{l}\text { track exploratory steps, allow } \\
\text { back-tracking }\end{array}$ & $\mathrm{X}$ & & & & $\mathrm{X}$ & & \\
\hline Extract & $\begin{array}{l}\text { save results of exploratory } \\
\text { steps }\end{array}$ & $\mathrm{X}$ & & & & $\mathrm{X}$ & & \\
\hline $\begin{array}{l}\text { Elaborate / } \\
\text { Abstract }\end{array}$ & show more or less detail & & & $\mathrm{X}$ & & & & $\mathrm{X}$ \\
\hline Select & mark something as interesting & & & $\mathrm{X}$ & $X$ & $X$ & & $\mathrm{X}$ \\
\hline Reconfigure & $\begin{array}{l}\text { change the arrangement, scale, } \\
\text { or encoding }\end{array}$ & & $\mathrm{X}$ & $\mathrm{X}$ & $\mathrm{X}$ & & & $\mathrm{X}$ \\
\hline Projection & & & $\mathrm{X}$ & & & & $\mathrm{X}$ & \\
\hline Distortion & & & $\mathrm{X}$ & & & & $\mathrm{X}$ & \\
\hline
\end{tabular}

often know what is available but do not always consider how individuals will use the system.

The study is based on a survey designed to simulate an interactive visualization displayed on a webpage. There was no preference given to the type of user (i.e., expert or novice). All responses were collected through an online survey system. The survey consisted of three pages of visualizations, with two questions about the data displayed in the interactive visualization.

\subsection{Tasks}

Tasks were selected based on the consideration that they should be presented in existing task taxonomies and used to evaluate the effectiveness of visualizations [23, 24]. Amar et al. (2005) proposed a taxonomy defining ten lower-level visual tasks describing the intent for using a visualization tool. These tasks are discussed in other task taxonomies. The ten visual tasks are described below [25].

Find Anomalies (FA) - identify any anomalies within a given set of data points concerning a given relationship or expectation.
Find Clusters (FC) - count the number of groups of similar data attribute values.

Compute Derived Value (CDV) - compute an aggregate value of data points.

Characterize Distribution (CD) - identify the distribution of that attribute's value over the set.

Find Extremum (FE) - find data points having an extreme value of a data attribute.

Order (ORD) - rank data points according to a specific ordinal metric.

Determine Range (DR) - find the span of values within a given set of data points and an attribute of interest.

Retrieve Value $(R V)$ - identify the values of attributes for given data points.

Filter (FTR) - find values satisfying a specific condition

Correlate (COR) - identify and determine the useful relationship between the values of attributes

\subsection{Visualization Design}


The visualizations used in this study were developed with the Google Charts application programming interface. Google Charts is a free service providing several types of charts, enabled with embedded interaction mechanisms. When deciding what chart types to include, we selected charts based on familiarity [23, 26]. The initial selection included bar charts, line charts, and table charts. This selection was finalized by consulting Stephen Few's Graph Selection Matrix [31]. The graph selection matrix states that line graphs are often used to show time series, deviation, and distribution. The data set used for the survey encompassed values that fit the need for a line chart as described by the graph selection matrix. Bar carts are often used to show ranking, part-towhole, deviations, and nominal comparisons. As with line charts, the dataset used also encompassed values that met this description. The decision of what type of chart to use for a visual representation came from information on the graph selection matrix coupled with the familiarity of line and bar charts. The development of the visual representations in terms of how to label axes, ink ratio and overall graphical clutter followed guidelines from Few [20], Tufte [27], and Ware [28].

Data sets may be complex and thus will need different types of analysis and visualizations to make sense of them. Two of the three visualizations included more than one type of chart. This design decision speaks to the flexibility of information visualization tools and differences in the cognitive processing of users [29].

\subsection{Interactions}

Embedded interactions incorporate one or more interactive graphical encoding into information visualizations [30]. The embedded interactions provided by Google Charts are subject to manipulation with the JavaScript programming language. All charts include details on demand, selection, highlighting, and distortion. The line chart includes a zoom feature, and the table chart includes a sort feature.

Embedded interactions do not have affordances to indicate their functional existence. Instructions were displayed in a yellow box below the visualization. We assumed that once an individual had used or been informed of the embedded interactions, instructions were not needed for future use. Instructions for selection and details on demand were provided on the first visualization and for zoom and sort on the second visualization. As all of the embedded interactions were available in the third visualization, no instructions were posted.

Embedded interactions change the display of the visual representation as a response to the trigger activated by the user. For example, the line chart responds to a click or selection of a data point by displaying a pop-up box, encoding the data point with a specified marker, and blurring out the rest of the chart's content (see Table 2).

Table 2. Embedded Interactions Matched to Tasks for Survey Visualization \#1

\begin{tabular}{|c|c|c|c|c|c|}
\hline Task(s) & $\begin{array}{l}\text { Interaction } \\
\text { Mechanism }\end{array}$ & Event & $\begin{array}{l}\text { Object of } \\
\text { Interest }\end{array}$ & $\begin{array}{l}\text { Embedded } \\
\text { Interaction }\end{array}$ & Example \\
\hline $\begin{array}{l}\text { Retrieve } \\
\text { Value } \\
\text { Find } \\
\text { Extremum }\end{array}$ & Select & Click & Data Point & $\begin{array}{l}\text { Encoding } \\
\text { Details on Demand } \\
\text { Focus + Context }\end{array}$ & $\begin{array}{l}\text { 211012, 12000 MM } \\
\text { sore } 12 \\
28.8 \mathrm{~K} \\
\end{array}$ \\
\hline \multirow[t]{3}{*}{$\begin{array}{l}\text { Find } \\
\text { Anomaly }\end{array}$} & Select & Click & $\begin{array}{l}\text { Data } \\
\text { Series }\end{array}$ & $\begin{array}{l}\text { Encoding } \\
\text { Focus + Context }\end{array}$ & \\
\hline & Select & Hover & Data Point & $\begin{array}{l}\text { Encoding } \\
\text { Details on Demand }\end{array}$ & $\begin{array}{l}2 / 10 / 12.12000 \mathrm{MM} \\
\text { stoes } 82 \\
28.8 \mathrm{~K}\end{array}$ \\
\hline & Select & Hover & $\begin{array}{l}\text { Data } \\
\text { Series }\end{array}$ & $\begin{array}{l}\text { Encoding } \\
\text { Highlighting }\end{array}$ & \\
\hline
\end{tabular}




\subsection{Procedure}

Each participant was asked questions related to the tasks defined in section 3.1. We matched these tasks to embedded interactions for each chart displayed using the granularity levels for human-information interaction. Table 2 provides a demonstration of how these elements come together to help decide which interaction mechanism supports a given task.

Select is a very common interaction mechanism that provides the ability to select something as interesting. We infer that select is carried out through two specific actions. The first action is clicking on an individual data point or a data series. Clicking directly on the data points causes the visualization to respond by encoding the data point with a special marker, display a pop-up box that provides additional information, and distort or blur the rest of the chart's content. The second action is hovering the mouse pointer over an individual data point or data series. Hovering over the data point will display the pop-up box with additional information and encode the data point with a special marker. Hover does not distort the rest of the chart's content. Table 2 provides visual examples of the embedded interactions and subsequent reactions for the selection mechanism.

\section{Analysis and results}

We distributed an online survey through email correspondence. We surveyed undergraduate and graduate students from two institutions, a rural private university, and a rural public university. We collected 129 complete surveys (74 percent of the respondents were male; 89 percent of all respondents were from the 18-24 age bracket). Of the participants, 16 percent use charts for daily or weekly activities; 50 percent use charts at least once a month; and 33 percent hardly user charts in their daily activities. Participants were not given training for how to use the system.

The online survey consisted of six questions relating to three visualizations. Each question has one correct answer, which was used to verify the survey results. The size questions were representative of a task or set of tasks. We used a scaffolding approach to design the visualizations and the survey questions. Table 2 provides the results of correct answers based on each question and visualization.
Table 3. Survey Results - Correct Answers

\begin{tabular}{|l|l|l|l|}
\hline Visualization & Question & Correct & Incorrect \\
\hline 1 & 1 & $76 \%$ & $24 \%$ \\
\hline 1 & 2 & $68 \%$ & $32 \%$ \\
\hline 2 & 3 & $88 \%$ & $12 \%$ \\
\hline 2 & 4 & $75 \%$ & $25 \%$ \\
\hline 3 & 5 & $92 \%$ & $8 \%$ \\
\hline 3 & 6 & $43 \%$ & $57 \%$ \\
\hline
\end{tabular}

The first visualization consisted of one line chart. The embedded interactions for this chart were selection by clicking and selection by hovering. Selection is the act of marking something as interesting [16]. Google Charts has four embedded interactions for selection. Selection by clicking a specific data point will encode that data point and blur out the rest of the context. The single data point is highlighted by a color and encoded with a special marker. Selection by clicking a specific data series will encode each data point along the series and blur out the rest of the chart content. Selection of a data series also occurs when the user clicks on an item in the legend (Figure 3-D). Hovering over a particular data point will display a pop-up box providing more details about the data point (Figure 3-B). Hovering over a particular item in the legend will highlight data series in the chart, but will not blur out the context of the graph (Figure 3-C).
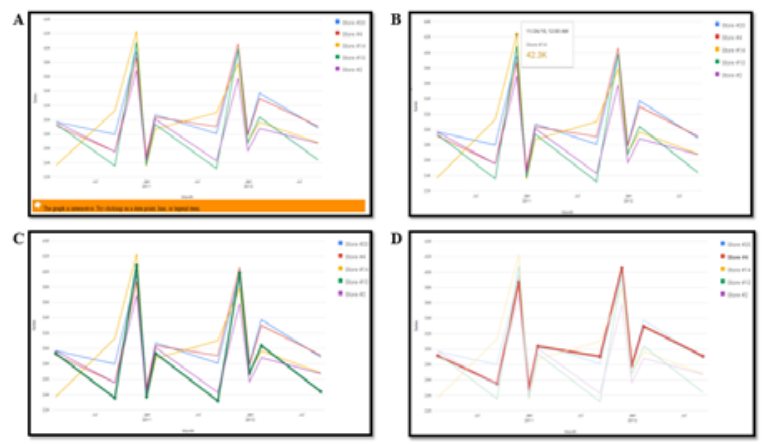

Figure 3. Visualization \#1. A) Original chart; B) Data point selection with details on demand; C) Data series selection with highlighting, and D) Data series selection with distortion.

All participants indicated that they used these embedded interactions to answer the questions. Participants were asked to find an extreme data point (FE) and retrieve its value (RV). They were also asked to determine the range of values (DR) and identify an anomaly (FA). The results of interacting with the first visualization provides initial evidence that selection supports tasks of finding extreme values, retrieving values, finding anomalies and determining the range of data values. The embedded interaction techniques for 
visualization one corresponding with the interaction techniques shown in Table 1 , specific select $[1,16,20$, $22]$ and details on demand $[1,16,18]$. More in depth testing is needed to identify the direct benefits for which embedded selection technique (clicking vs. hovering) is more supportive of the given task(s).

Table 4 figure A provide insight to how participants used interaction mechanisms to complete the given task. The chart demonstrates what interaction was used based on if the question was answered correctly or incorrectly. The majority of participants did get this question correct, and also identified that the selection (whether by clicking or hovering) interaction mechanisms were instrumental in answering the question. We see that the selection interactions did assist the majority of participants in answering the questions. There were a number of participants with incorrect answers that claimed to use the selection mechanisms. More direct observation is needed to understand how these individuals fully used the interactive visualization.

The second visualization consisted of one line chart and one table chart. The embedded interactions for the line chart were selection by clicking, selection by hovering, and filter by zooming. The embedded interaction for the table chart was sorting. Participants were asked to complete two tasks: 1 ) retrieve a value after sorting the data (ORD); and 2) to compute a value based on the displayed data (CDV). The charts on visualization \#2 supported the selection interaction mechanisms found on visualization \#1. We used two additional embedded interactions for the second visualization, zoom and sort. By clicking anywhere on the line graph and dragging, users were able to zoom into a particular region (Figure 4-C). This action relates to the abstraction or elaboration interaction technique specified in Table 1. With the table chart, the user could click on the header of a row and sort the table in ascending order (Figure 4-D). If they clicked a second time on the header the data would be sorted in descending order.

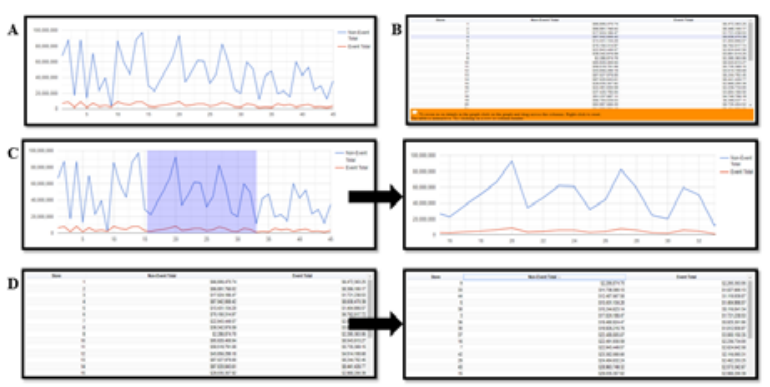

Figure 4. Visualization \#2. A) Original line chart; B) Original table chart; C) Line chart with zoom and D) Table chart with sort.
Participants indicated that they used sorting, selection by clicking and selection by hovering. The majority of participants did not use the zoom feature or found it unhelpful for completing tasks. The results of interacting with the second visualization provides evidence for how selection and ordering interaction mechanisms support tasks for computing derived values and ordering values. For tasks relating to ordering values, the participants identified the sort mechanism as being useful. For tasks relating to calculating a derived value, participants identified the hover-selection mechanism as being useful. Following the framework of HII, these results also suggest that sub-tasks are necessary to complete the overall intent. For example, sorting the values is needed before a value can be found or retrieved. The zoom feature was not used to complete the tasks, therefore we have insufficient evidence to the utility of this embedded interaction mechanism for low-level analysis.

Table 4 figures $\mathrm{B}$ through $\mathrm{E}$ provide insight to how participants used interaction mechanisms to complete the given task. The charts show the number of participants that used the interaction mechanisms based on if they got the question correct or not.

The third visualization consisted of one bar chart and one table chart. The embedded interactions for the bar chart were selection by clicking and selection by hovering. Selecting a specific bar or data point in the chart highlighted the data series and blurred out the rest of the charts context (Figure 5-B). As with the line chart, hovering over a specific data value would display a pop-up box providing more information about the data point. The embedded interaction for the table chart was sorting (Figure 5-C).

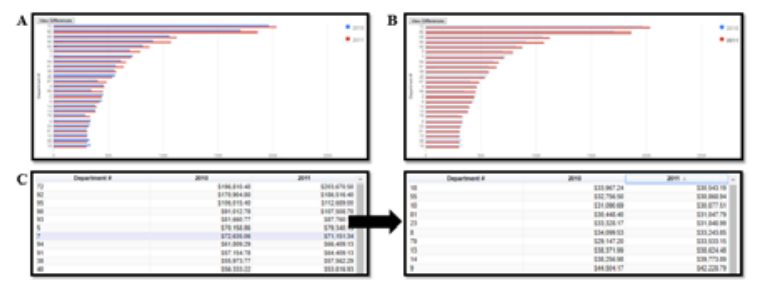

Figure 5. Visualization \#3. A) Original bar chart; B) Data series with selection distortion and $C$ ) table chart with sort.

Participants were asked to identify a cluster of data values (FC) and to characterize the distribution of values (CD). The majority of participants indicated they used the sorting feature to answer the questions, while there was some reliance on selection by clicking or hovering. The results of interacting with the third interaction provides evidence for how selection and ordering interaction mechanisms supports the tasks of clustering, determine the range of values, and 
characterizing the distribution. Participants relied on the selection by hovering for clustering and determining the range of values, whereas they relied on sorting to characterize the distribution of values.

Table 4 figures $\mathrm{F}$ through $\mathrm{H}$ provide insight to how participants used interaction mechanisms to complete the tasks of finding clusters, determining the range of values, and characterizing the distribution.

\subsection{Design recommendations}

Selection interaction mechanisms are important for supporting low-level analysis tasks. Designers should look for ways to incorporate these mechanism, whether the action of clicking changes the visual representation or the action of hovering displays details on demand.

The ability to sort values in a table chart are important for supporting the low-level task of order. The table chart may apply ascending or descending order for a selected column. The act of sorting can be seen as a way to reconfigure the data values, as identified as an interaction mechanism by [16, 19, 20, 22]. More testing is needed to determine if sorting data values should be applied to more than just the table chart. The connection of interaction mechanisms between different visualizations is called linking [5], which is not explicitly tested in this study.

The design assumption that users will remember and utilize embedded interactions each time a visualization is encountered appears to be misleading. The scaffolding attempt of displaying interaction instructions just once and assuming these would be remembered at a later date was ill-advised.

In summary, we identify the following aspects that interactive visualization designers should consider:

- Implement selection interaction mechanisms to support the low-level analysis tasks of find anomalies, find extremum, and retrieve value.

- Implement sorting interaction mechanisms to support the low-level analysis task of order.

- Interaction mechanisms for selection may support sub-tasks to the low-level tasks of clustering, computing derived values, ordering, and characterizing distribution.

- The functional existence of embedded interactions must be explicitly stated on each and every visualization.

These results support extant research demonstrating the use of interactivity to support analysis tasks. Evaluation of interactive visualizations remains a complex challenge within the discipline. The diversity of when and how users utilize information visualizations, coupled with the myriad of options for visual representations and interaction mechanisms creates challenges for designers to know what to implement.

\section{Limitations and future work}

This research was a pilot study to gather information about which interaction mechanisms are used when individuals find an interactive visualization on the Internet. Results provide information that interactions were used to support analysis tasks, but did not provide sufficient evidence to measure effectiveness. Further testing is needed through observations or event tracking on websites to provide further evidence of interaction benefits.

The results of this study support extant research in that interaction mechanisms provide cognitive aids to individuals using visualization systems. The benefits of interaction mechanisms are yet to be fully defined, but it is clear that designers of visualization systems need to understand the coupling between interactions and visual representations.

We provide initial evidence that interactions do benefit analysis tasks. Our results show that the interaction mechanisms for selection support the lower-level tasks to finding extreme values, retrieving values, finding anomalies, and determining the range of values. Visual reactions that users see while working with select include highlighting the data series, encoding the data value selected, providing a tooltip with additional details about the selected data point, and focusing on the selected data point while distorting other data displayed in the chart. Visual designers need to be aware of this benefit and ensure the interaction and subsequent reactions are embedded in information visualizations.

The next steps for this research is to delve deeper into clearly defining interaction mechanisms. For instance, select is defined as marking something of interests [16]. The action of select can be interpreted differently. The Google Charts API allows select to occur by clicking which encodes the data point and blurs or distorts the rest of the chart, by hovering encoding the data point and displaying a pop-up box, and by highlighting which brings to focus the data series while the rest of the chart is blurred or distorted. Our research shows that embedded mechanisms support at best eight of the ten lower-level analysis tasks. Controls external to the chart itself will need to be implemented to fully realize the benefits of interactions for tasks that involve filtering and correlation. Our goal is to take the data from this pilot study and carry it forward to more conclusive testing by observing users with online interactive visualizations that include external controls and embedded interactions. 
A

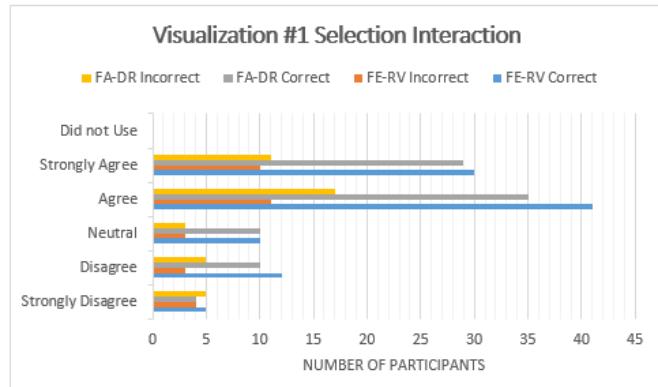

C

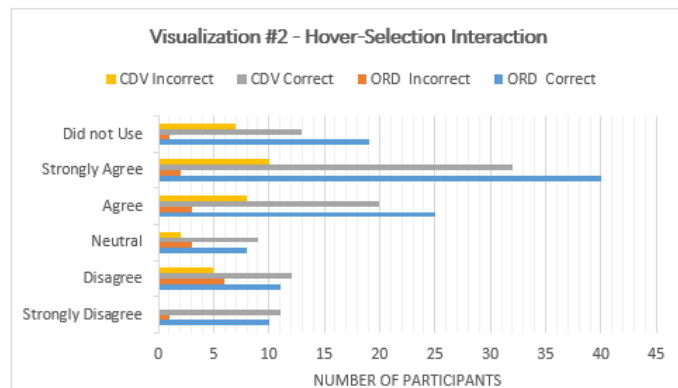

$\mathbf{E}$

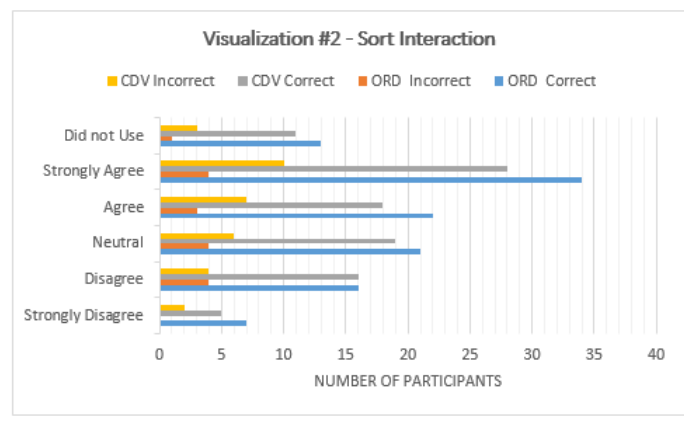

G

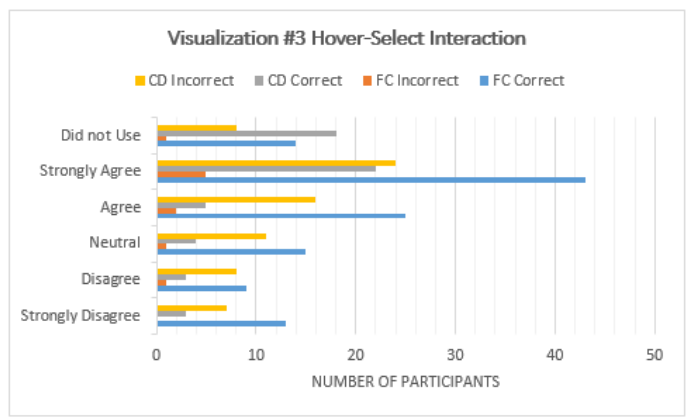

\section{Conclusion}

Information visualizations are a cognitive tool, much like a pencil or a calculator. People with cognitive tools are more effective thinkers than people without. Before the efficiency of a tool can be measured, we must understand what aspects of that tool are being used. This study offers insight into how
B

\section{D}

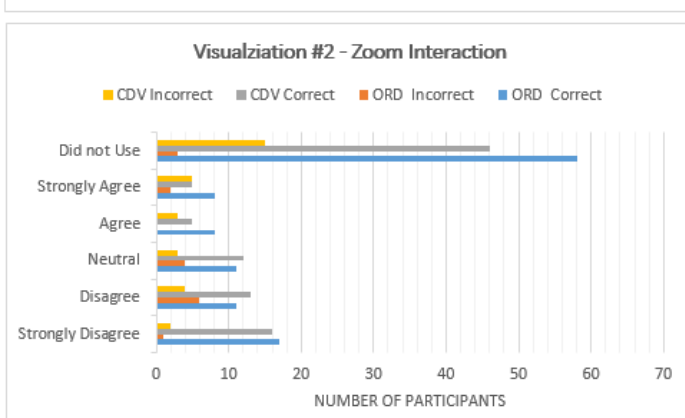

$\mathbf{F}$

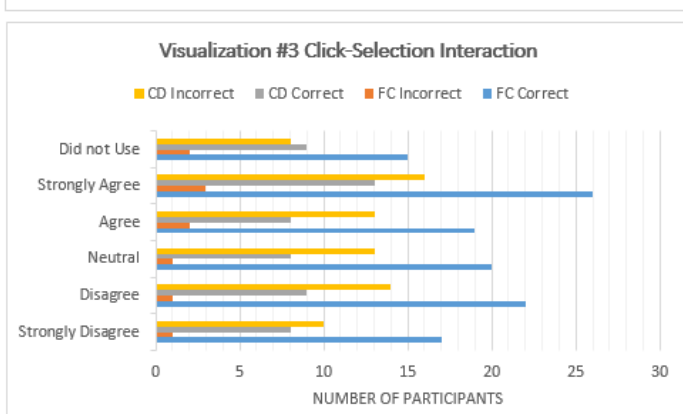

H

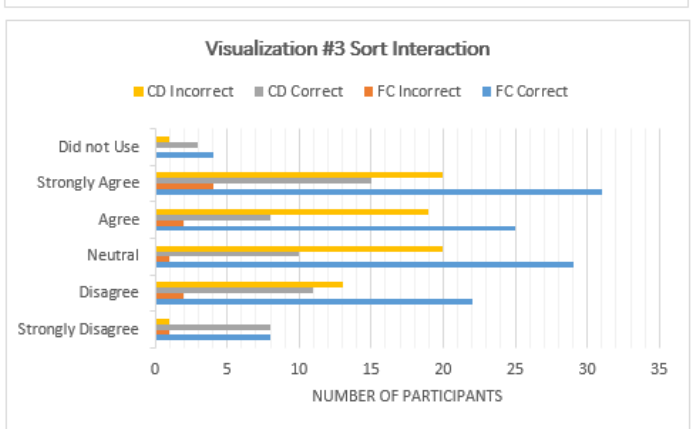

users interact with visualizations that they find online. It also provides a starting point to further develop how interactive mechanisms can be mapped to analysis tasks.

\section{References}

[1] J. Heer, \& B. Shneiderman. "Interactive dynamics for visual analytics”, Queue, 2012 10(2), pp. 30. 
[2] P. Parsons, K. Sedig, A. Didandeh, \& A. Khosravi, "Interactivity in visual analytics: use of conceptual frameworks to support human-centered design of a decisionsupport tool.” Paper presented at the System Sciences (HICSS), 2015, 48 ${ }^{\text {th }}$ Hawaii International Conference.

[3] Card, S.K., J.D. Mackinlay, \& B. Shneiderman, Readings in information visualization: using vision to think: Morgan Kaufmann Publishers; 1999.

[4] P. Parsons \& K. Sedig, “Adjustable properties of visual representations: Improving the quality of human-information interaction." Journal of the Association for Information Science and Technology, 2014, 65(3), pp.455-482.

[5] Ward, M., G. Grinstein, \& D. Keim. Interactive Data visualizations: Foundations, Techniques and Appliations (Second Edition), Taylor \& Francis Group, Boca Raton, FL, 2015.

[6] E.H. Chi, \& J.T. Riedl, “An operator interaction framework for visualization systems.” Paper presented at IEEE Symposium on Information Visualization. Oct. 1998, p. 63-70.

[7] R.H. Kosara \& D.L. Gresh, “An interaction view on information visualization”, Proceedings of EUROGRAPHICS, 2003, p. 123-137.

[8] M.A. Yalcin, N. Elmqvist, \& B.B. Bederson, "Cognitive stages in visual data exploration", Paper presented at the Proceedings of the Sixth Workshop on Beyond Time and Errors on Novel Evaluation Methods for Visualization, ACM, October 24 2016, p. 86-95.

[9] W. Aigner. "Understanding the role and value of interaction: First Steps.” The Eurographics Association. Paper presented at the Proceedings of the International Workshop on Visual Analytics, 2011, p. 17-20.

[10]. K. Sedig, P. Parsons, M. Dittmer, \& R. Haworth. "Human-centered interactivity of visualization tools: Microand macro-level considerations." Handbook of Human Centric Visualization. Springer. 2014, p. 717-743

[11] R.E. Roth, “An empirically-derived taxonomy of interaction primitives for interactive cartography and geovisualization”, IEEE, Transactions on Visualization and Computer Graphics, 2013, 19(2), p. 2356-2365.

[12] Spence, R. Information Visualization: Design for Interaction (Second Edition). Pearson. Harlow, England. 2007.

[13] K. Sedig, P. Parsons, H.N. Liang, \& J. Morey. "Supporting Sensemaking of complex objects with visualizations: Visibility and complementarity of interactions.” Informatics. Multidisciplinary Digital Publishing Institute. 2016 4(3), p. 20.
[14] J.J. Van wijk. "The value of visualization." IEEE, Visualization. 2005 p. 79-86.

[15] B. Lee, P. Isenberg, N. Henry-Riche, \& S. Carpendale. "Beyond mouse and keyboard: Expanding design considerations for information visualization interactions”, IEEE, Transactions on Visualization and Computer Graphics, 2012, 18(2), p. 2689-2698.

[16] J.S. Yi, Y. Kang, \& J. Stasko. "Toward a Deeper Understanding of the Role of Interaction in Information Visualization”, IEEE, Transactions on Visualization and Computer Graphics, 2007, 13(6), p. 1224-1231.

[17] W.A. Pike, J. Stasko, R. Chang, \& T.A. O’Connell. "The Science of Interaction", Information Visualization, 2009, 8(4), p. 263-274.

[18] B. Shneiderman, "The eyes have it: a task by data type taxonomy for information visualization.” IEEE, Proceedings 1996 IEEE symposium on visual languages, 1996, p. 336343.

[19] D.A. Keim, "Information visualization and data mining”, IEEE, Transactions on Visualization and Computer Graphics, 2002, 8(1), p. 1-8.

[20] Few, S. Now You See It: Simple Visualization Techniques for Quantitative Analysis. Analytics Press. 2009.

[21] Borner, K. Atlas of Knowledge: Anyone Can Map. The MIT Press. Cambridge, Massachusetts. 2015.

[22] A. Figueiras, "Towards the understanding of interaction in information visualization”, IEEE, Paper presented at the Information Visualization $19^{\text {th }}$ International Conference, July 2015, p. 140-147.

[23] B. Saket, A. Endert, \& C. Demiralp. "Task-based effectiveness of basic visualizations," IEEE, Transactions on Visualization and Computer Graphics, 2018, 25(7), p. 25052512.

[24] K. Sedig, \& P. Parsons, "Interaction design for complex cognitive activities with visual representations: A patternbased approach”, AIS Transactions on Human-Computer Interaction, 2013, 52(2), p.84-133.

[25]. R. Amar, J. Eagan, \& J. Stasko. "Low-level components of analytic activity in information visualization", IEEE, Symposium on Information Visualization, 2005, p. 111-117.

[26] W.S. Cleveland \& R. McGill, "Graphical perception: Theory, experimentation, and application to the development of graphical methods", Journal of the American Statistical Association, 1984, 79(387), p. 531-554.

[27] Tufte, E.R., The visual display of quantitative information., Graphics Press, Cheshire, CT. 2001.

[28] Ware, C. Information visualization: perception for design. Morgan Kaufmann, Boston, 2012. 
[29] Munzner, T. Visualization analysis and design. CRC Press, Boca Raton, FL. 2015.

[30] B. Saket, H. Kim, E.T., Brown \& A. Endert. "Visualization by Demonstration: An Interaction Paradigm for Visual Data Exploration." IEEE, Transactions on Visualization and Computer Grpahics, 2017, 23(1), p. 331340.
[31] Few, S. Show Me the Numbers: Designing Tables and Graphs to Enlighten (Second Edition). Analytics Press. Burlingame, CA. 2012. 